\title{
Les Perspectives d'Evolution de l'Assurance Vie en France
}

\author{
par Guy Verdeil *
}

L'assurance vie, après avoir connu des brillants développements avant 1914, n'a repris en France de dévelopement significatif que dans les années 1960.

Une longue période de stagnation a donc contribué à réduire son rôle économique et social. Les primes d'assurance vie ne représentent en France que $1 \%$ du PNB, contre 3,4 \% au Japon, $3 \%$ en Grande-Bretagne, 2,4 \% aux Etats-Unis, $2 \%$ en RFA, soit un niveau de $450 \mathrm{~F}$ par tête sensiblement inférieur à celui de nos grands partenaires commerciaux.

Ce retard relatif est la conséquence de données propres à l'évolution économique de la France, et notamment de la perméabilité particulière de ce pays au développement d'une inflation en général plus élevée sur une longue période que dans les grands pays. Il tient aussi à la vigueur et à l'extension des systèmes de couverture sociale obligatoire (allocations familiales, sécurité sociale et systèmes de retraites) depuis les années 1930 et suivantes : dans ce domaine, l'impuissance relative de l'assurance à compenser les effets de l'inflation a d'ailleurs contribué, surtout après la deuxième guerre mondiale, à renforcer les systèmes obligatoires reposant sur le principe de la répartition.

La croissance économique de la décennie 1960, la relative modération de l'inflation et la reconnaissance par les pouvoirs publics à travers diverses déductions fiscales du rôle de l'assurance vie comme canal de création d'une épargne utile au développement économique, ont permis à l'assurance vie de retrouver les voies d'un développement plus rapide que celui de la production nationale. Le ralentissement de la croissance et l'accélération de l'inflation qui ont caractérisé l'évolution économique depuis une dizaine d'années n'ont pas contrarié ce mouvement. Aujourd'hui comme hier, l'évolution du taux d'inflation et du champ couvert par les régimes sociaux obligatoires conditionnent pour l'essentiel l'avenir de l'assurance vie en France. Mais, ces deux facteurs agiront sans doute davantage sur sa structure et ses modes de gestion que sur son développement auquel s'ouvrent au contraire des chances nouvelles.

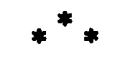

L'assurance vie restera largement tributaire à l'avenir de l'évolution des politiques suivies en matière de lutte contre l'inflation et de taux d'intérêt.

* Président, Groupe des Assurances Nationales, Paris. 
Pour sa part, l'assurance vie en France a su s'adapter dans sa politique de produits et sa gestion à la nécessité de satisfaire un consommateur de plus en plus conscient des effets de l'inflation sur son épargne et sur ses revenus. Elle a su, de ce fait, résister aux effets de l'accélération mondiale des taux d'inflation depuis 1970-1971, puis après les chocs pétroliers, en lançant des contrats indexés sur des unités de compte et en développant la technique de la participation des assurés aux bénéfices d'une gestion financière des placements qui a profité de la hausse des taux d'intérêts des obligations depuis quelques années.

Cette adaptation a toutefois une contrepartie : elle a contribué à une assimilation sans doute excessive des produits d'assurance à des produits d'épargne et de placements dans l'esprit du public, et l'ont, de ce fait, rendu plus sensible à l'évolution des comportements d'épargne des ménages comme à la concurrence des autres institutions financières et des banques pour une part croissante de son activité.

L'expérience des dernières années montre que ce sont surtout les fortes variations du taux d'inflation, plus que son niveau absolu, qui ont le plus sensibilisé le public à l'évolution du rendement de ses actifs épargnés et ont exercé la plus forte pression sur le comportement des compagnies d'assurances et la modification de leurs produits.

Trois facteurs pourraient commander l'évolution des prochaines années :

- Les perspectives de croissance ralentie, qui sont à moyen terme celles de la plupart des grands pays industrialisés, devraient maintenir la tendance à la baisse du taux d'épargne des ménages qui ajustent de plus en plus leur épargne financière à la rentabilité des produits offerts. On observe en effet une tendance régulière en France depuis 1980 : une baisse du taux d'épargne de plus d'un demi-point par an, ce qui en fait un phénomène économique non négligeable. Mais, à l'intérieur de cet ensemble, c'est l'épargne affectée à l'investissement en logement qui supporte cette baisse, tandis que se maintient l'épargne de précaution et que progresse surtout l'épargne financière.

- Les besoins d'épargne longue liés au financement de la restructuration industrielle en cours dans tous les grands pays sont normalement appelés à s'accroître et devraient se traduire par le maintien de taux d'intérêt relativement élevés par rapport aux taux d'inflation. Il en ira d'autant plus ainsi que les grandes nations industrielles connaissent des déficits publics importants, développés en compensation de politiques de stabilisation particulièrement sévères depuis le $2^{\mathrm{e}}$ choc pétrolier, pour financer des dépenses d'assurance chômage, des prestations sociales, des grands travaux ou le soutien à de grandes entreprises en difficultés. Il est peu probable l'exemple des Etats-Unis de M. Reagan le montre clairement - que ces déficits puissent être rapidement résorbés; ils viendront donc pendant longtemps encore s'ajouter aux besoins des entreprises, pour exercer une pression forte à la hausse de la demande de capitaux à long terme, même si une entreprise économique et des politiques de rigueur budgétaire et fiscales viennent progressivement l'alléger. A cet égard, la France n'a que peu de chances de se distinguer du peloton.

- Enfin, en France, plus qu'ailleurs, les banques, gênées par la hausse du coût de leurs ressources et par la gratuité du chèque, sont à la recherche de sources d'alimentation rentables de leur chiffre d'affaires à l'avenir et sont intéressées par le marché des produits d'assurance, les plus proches des produits d'épargne. Elles ont déjà 
tenté de la faire avec succès dans le domaine des «bons de capitalisation » et de produits bénéficiant des avantages fiscaux de l'assurance vie qu'elles vendront à leurs guichets avec des chargements commerciaux très réduits.

Il y a là, pour l'assurance, un aiguillon, mais aussi un risque d'autant plus fort que ses coûts de gestion - essentiellement en personnel de distribution ou de gestion proprement dite, de contrats en général plus complexes que de simples produits d'épargne - sont stimulés eux aussi par l'inflation.

Deux réponses doivent donc être combinées par les sociétés d'assurance sur la vie et le sont en effet :

- Obtenir des gains de ces coûts, notamment par une automatisation accrue : c'est l'objet des efforts accomplis actuellement par de nombreuses compagnies d'assurances pour développer leur gestion informatique en temps réel et faciliter le développement de l'utilisation par leurs réseaux commerciaux salariés ou mandataires - d'outils automatisés de gestion ou de support commercial : il y a là pour le personnel et les réseaux des compagnies d'assurances une source de transformation considérable de leurs conditions traditionnelles de travail.

- Accroître la redistribution aux assurés, quitte, et certains le regrettent, à rompre la solidarité entre les générations en isolant - ou cantonnant - certains des actifs au profit des nouveaux clients. C'est là sans doute la transformation qui pose le plus de problèmes «stratégiques » aux assureurs français. Au-delà du débat, sans doute un peu formel et que les faits trancheront, sur le problème de savoir si l'on doit ou non assimiler l'assurance vie à un produit financier, les compagnies d'assurances (et derrière elles les pouvoirs publics et les consommateurs) seront peut-être amenés à faire un choix devant lequel elles reculent actuellement en prenant des mesures « conservatoires ». Pour faire face à la concurrence de produits très proches des produits financiers, vendus avec de très faibles frais de commercialisation, les compagnies traditionnelles créent et lancent des produits du même type en isolant les actifs - immobiliers ou obligataires - qui gagent l'épargne recueillie. Ces produits se vendent bien, puisque le «cantonnement 》 ou la spécialisation des placements permet aux compagnies de faire bénéficier leur nouvelle clientèle des taux d'intérêt exceptionnels atteints ces dernières années par les obligations ou des plus-values sur des actifs immobiliers particulièrement sélectionnés. Aussi longtemps que la part du marché total de l'assurance vie prise par ces produits reste limitée, la solidarité et l'égalité de traitement entre assurés anciens et assurés nouveaux ne sont pas excessivement remises en cause. Les grandes compagnies en particulier, qui disposent chaque année de flux importants de provisions nouvelles à placer aux taux les plus rémunérateurs, n'ont pas trop de difficultés à élever de manière sensible le taux moyen de rendement de l'ensemble de leur portefeuille et à revaloriser leurs contrats anciens.

Il en irait autrement si la pression concurrentielle qui s'exerce aujourd'hui sur ces compagnies de la part d'entreprises nouvelles - sans clientèle ancienne et sans historique - devait s'accroître ; il en résulterait normalement soit une profonde crise dans les modes de distribution de l'assurance et une intégralité entre classes d'âge d'assurés préjudiciable à la collectivité, soit sans doute un réexamen et une différenciation dans l'utilisation de l'incitation fiscale. 
L'mtensité de la pression qui s'exercera ainsi sur les compagnies d'assurances dépend finalement non seulement du niveau et de la stabilité du taux d'mflation ou des déterminants à long terme de l'épargne, mais aussi largement du rapport qui s'établira à l'avenir entre le taux d'intérêt à long terme et le taux d'inflation. Le maintien d'un écart positif au profit du taux d'imtérêt lié à un ralentissement du taux d'inflation demeurera la situation la plus favorable du développement harmonieux de l'assurance vie. Le retour à des taux d'inflation élevés du type de ceux que le monde occidental a connus de 1978 à 1983 resterait un sérieux facteur de remise en cause dans les structures actuelles de l'industrie de l'assurance vie.

L'hypothèse du " parallélisme des taux » est-elle vraisemblable ? L'évolution des besoins de financement longs dans le monde peut le faire penser. A l'mverse, les difficultés de la reconstitution d'une discipline monétaire imternationale pourraient conduire pendant quelques années le monde occidental à osciller de reprises avortées en stagnations prolongées et entraîner un cloisonnement des économies, fatal à la croissance mais non à l'inflation. Un scénario de crise et d'isolement ramènerait alors sûrement l'assurance à ses plus mauvaises années de l'immédiate après-guerre, où se conjugueraient l'mflation, un faible croissance et de bas taux d'intérêt.

Si les facteurs économiques du développement de l'assurance vie restent aussi incertains, car liés à des évolutions macro-économiques internationales difficilement prévisibles, l'évolution des facteurs institutionnels paraît ouvrir des perspectives nouvelles intéressantes et prochames à l'assurance vie en France : celui du marché de la retraite par capitalisation.

L'évolution des systèmes de retraites en France s'inscrit dans le contexte d'ensemble, spécifique à la France, de l'évolution du financement des systèmes de couverture sociale et encore plus largement dans l'évolution à long terme de l'économie tout entière. Plusieurs éléments convergent, de ce fait, pour élargir un marché resté jusqu'alors plus limité qu'à l'étranger à l'assurance vie en France :

- difficultés, appelées à s'accroître, des régimes de retraite par répartition ;

- la réhabilitation des techniques de la capitalisation;

- l'intérêt manifesté par les pouvoirs publics à des mécanismes de développement d'une épargne longue nouvelle sous la forme de la constitution de retraites par capitalisation.

Sur le premier point, ce ne sont pas essentiellement les facteurs démographiques - comme le croit souvent le grand public - qui menacent, au moins à court terme, le financement des régimes actuels, basés sur la répartition - mais essentiellement des facteurs institutionnels au regard de l'évolution économique générale. Ceux-ci proviennent des nombreuses mnovations introduites dans le droit social et qui toutes se traduisent par un alourdissement des charges financières de prestations (garanties de ressources et avancement de l'âge de la retraite) alors que l'aggravation du chômage vient limiter les ressources et que, sur un plan plus général, la faible croissance de l'économie rend insupportable aux entreprises et dans une certame mesure aux 
ménages l'alourdissement de l'ensemble des prélèvements obligatoires qu'elles subissent les uns et les autres, pour financer l'ensemble de la couverture sociale. Les études préparatoires au $\mathrm{IX}^{\mathrm{e}}$ Plan ont bien mis en lumière l'incompatibilité à terme d'une croissance faible de l'économie, d'un besoin de financement par les entreprises d'un effort important d'investissement, d'une limitation de la croissance des charges salariales des entreprises, avec la poursuite d'un financement des régimes de couvertures sociales prolongeant les tendances passées. Les pouvoirs publics s'assignent pour objectif de stabiliser le prélèvement obligatoire total subi par les entreprises au niveau atteint aujourd'hui. C'est dans cette perspective qu'est entreprise une politique de maîtrise des dépenses qui touche tous les domaines de la couverture sociale, maladie, hospitalisation, aide aux familles, allocations chômage et non les seules retraites. L'idée d'un transfert de certaines de ces charges vers les ménages, à travers soit un financement fiscal (familles), soit une participation individualisée, se développe.

Déjà, certains régimes complémentaires ont vu la valeur de leurs prestations exprimées en " points » de retraite - se réduire depuis quelques années.

Dans ce contexte, les régimes obligatoires de répartition existants ne peuvent espérer voir leurs ressources se développer au rythme de leurs besoins définis par l'attente d'une revalorisation régulière des retraites servies, sauf à admettre qu'une partie des besoins de couverture ressentie par le public pourra s'exprimer à travers des systèmes volontaires de retraites par capitalisation. Ainsi la retraite, qui s'est définie longtemps en France comme un transfert de ressources entre générations, est-elle appelée à prendre une forme mixte, où au transfert s'ajoutera un étage d'épargne.

Or, la méfiance ancienne à l'égard des mécanismes de la capitalisation - individuelle ou « collective » à travers des groupes mutualisés de formes diverses - s'estompe aujourd'hui à l'analyse. D'une part, les produits nouveaux d'assurance garantissent beaucoup mieux l'épargnant contre l'inflation, comme on l'a vu plus haut. Il apparaît bien, d'autre part, qu'il s'agirait d'un apport complémentaire, ce qui réduit de beaucoup la portée des calculs théoriques présentés dans le passé par certains experts dans une hypothèse de substitution de la capitalisation à la répartition - et qui montraient l'impossibilité de cette substitution à raison de la masse des réserves nécessaires.

De nombreuses réflexions - menées notamment à l'occasion du IXe Plan s'orientent vers des formes de gestion collective, du type de l'assurance de groupe ou de plans d'épargne d'entreprise, d'une épargne destinée en quelque sorte à préfinancer un niveau additionnel de ressources de retraite. L'assurance connaît bien ces mécanismes, dont l'efficacité sera toutefois liée aux possibilités contributives des entreprises et à l'évolution de relations contractuelles entre celles-ci et leurs salariés. Hors du monde du salariat, l'assurance Grandes Branches classique a toutes ses chances et répond déjà à des besoins en croissance.

En revanche, les procédures individuelles de capitalisation conduisent effectivement à une inégalité des pensions de retraite sensiblement plus forte que celle qui découle d'une procédure de répartition. Comme le note un des rapports rédigés à l'intention de la Commission Dautresme, « l'inégalité des pensions de retraite issues de la répartition provient principalement de l'inégalité des revenus d'activité, alors que l'inégalité des rentes de capitalisation intègre aussi les différences de taux d'épargne ». 
Quelle que soit la forme choisie - gestion dite « collective» ou gestion dite * individuelle » - de l'effort sur-complémentaire de constitution de retraite par capitalisation, celui-ci entraîne sinon toujours une création nette d'épargne, du moins un déplacement de l'épargne vers des durées longues d'immobilisation qui correspondent bien aux besoins de consolidation du financement de l'important effort de restructuration de l'appareil productif envisagé par le IX $\mathrm{X}^{\mathrm{e}} \mathrm{Plan}$. Il y a là encore une chance pour l'assurance vie, particulièrement armée pour gérer des engagements de longue durée et pour procéder à des placements importants, mais diversifiés et sûrs.

Dans un environnement économique incertain, mais où les besoins d'épargne longue sont appelés à l'accroître, face à une clientèle plus exigeante sur la transparence et la rentabilité des produits, mais désireuse d'explorer de nouveaux mécanismes de protection sociale, l'assurance vie paraît avoir en France des perspectives de développement d'autant plus prometteuses que la puissance publique veillera à maintenir des règles de concurrence loyale avec les autres systèmes de collecte de l'épargne et de garantie des risques sociaux. 